\title{
3-D TRANSIENT SIMULATION OF A FLAT CAPPILARY HEAT PIPE VIA AN INTERFACE TRACKING METHOD
}

\author{
H. A. Machado \\ Centro Técnico Aeroespacial - CTA \\ Instituto de Aeronáutica e Espaço - IAE \\ Pr. Mal Eduardo Gomes, 50, Vila das Acácias \\ 12228-904, São José dos Campos, SP, Brasi \\ humbertoam@iae.cta.b \\ Universidade do Estado do Rio de Janeiro \\ Faculdade de Tecnologia de Resende \\ Estrada Resende-Riachuelo, s/n, Morada da \\ Colina \\ 27523-000, Resende, RJ, Brasil \\ machado@fat.uerj.br

\section{NOMENCLATURE} \\ c specific heat, J/kg.K \\ $\mathrm{G}$ gravity, $\mathrm{m} / \mathrm{s}^{2}$ \\ I indicator function \\ $\mathrm{K}$ thermal conductivity, W/m.K \\ $\mathrm{L} \quad$ latent heat of phase change, $\mathrm{J} / \mathrm{kg}$ \\ $\mathrm{P}$ pressure, $\mathrm{Pa}$ \\ Pr Prandtl number, $\operatorname{Pr}=v / \alpha$ \\ $\mathrm{t}$ time, $\mathrm{s}$ \\ $\mathrm{T}$ temperature \\ u velocity vector, $\mathrm{m} / \mathrm{s}$ \\ $\mathrm{u}, \mathrm{v}, \mathrm{w}$ velocity components, $\mathrm{m} / \mathrm{s}$ \\ $\mathbf{x} \quad$ position vector, $\mathrm{m}$ \\ $\mathrm{x}, \mathrm{y}, \mathrm{z}$ Cartesian coordinates, $\mathrm{m}$
}

\section{Greek symbols}

$\begin{array}{ll}\alpha & \text { thermal diffusivity, } \mathrm{m}^{2} / \mathrm{s} \\ \rho & \text { density, } \mathrm{kg} / \mathrm{m}^{3} \\ \mu & \text { viscosity, Pa.s } \\ \mathrm{w} & \text { relaxation coefficient }\end{array}$

\section{Subscripts}

f interface

1 liquid phase

v vapor phase

0 reference or initial value

SAT saturation condition

$\infty \quad$ free stream

\section{ABSTRACT}

In capillary and micro heat pipes the internal porous media is replaced by a capillary groove, yielding a meniscus formed by the liquid phase. The meniscus height varies along the groove length, from the condenser section to the evaporator section, and the difference between the pressures in each extremity is responsible for pumping the liquid. Such devices have been employed in electronic cooling, due its small dimensions. This system provides excellent thermal control, assuring uniform temperature distribution. In previous works related to capillary heat pipe simulation, empirical or simple 1-D models have been used, but not taking into account the complete unsteady phenomena. In this work, the 3-D unsteady simulation of a flat micro heat pipe is presented, where the meniscus radius is considered constant along the transversal section. The phase change problem of a heated liquid meniscus in a groove is simulated via an interface tracking method, which is an hybrid Lagrangean-Eulerian method for moving boundary problems. A variation of the one-dimensional Stephan problem is used to validate the numerical code.

Keywords: Capillary and micro heat pipes, Moving boundary, Phase change, Interface tracking

\section{INTRODUCTION}

Phase change processes through capillary grooves are commonly incorporated in design of heat exchangers, increasing the interfacial areas among liquid-vapor phases for evaporation and vapor-liquid phases for condensation. These grooves allow the liquid phase to coalesce and move from the condensation region for the evaporator, promoting the reduction of thickness of the liquid film. Recently, the use of such systems in micro scale devices for cooling of electronic equipment has deserved attention, due its high heat transfer rates and excellent thermal control, improving the uniformity of temperature distribution, specially when employed as micro heat pipes, that are built straight over the chips structure. Mini and micro heat pipes have been employed in high performance computers, laptops and workstations, satellites and space probes, fine surgery instruments, etc.

The development of this kind of heat pipe has been done using empirical methods or simplified models. Most of the experimental works deal with a specific aspect of micro heat pipes, such as a particular shape or phenomenon involved in the pipe operation, or accuracy of any theoretical approximation used in the model. In these cases, some information is available about thermocappilary effects in a heated meniscus (Prat et al, 1998) or about the influence of geometry in the heat exchange process (Ravigururajan, 1998). In all experiments, several difficulties were found in reproducing the realistic dimensions and conditions of operation of a 
feasible M.H.P (Faghri, 1995). Huang et al (1998) studied a constrained vapor bubble behavior, and characterized it as a large version of a micro heat pipe. A strong dependence of its performance with the orientation with respect to gravity was observed.

Sun \& Tien (1972) presented a simple conduction model for steady state operation with an enclosure condition, based in the local wall temperature and the mass flow rate, and compared with some experimental data available in literature. The results demonstrated the high heat transfer capacity of such devices, and its dependence to Biot number and relative contribution of radial conduction through the wick and axial conduction to the pipe wall.

Duncan and Peterson (1994) summarized the conclusions and limitations of each model available in a opened literature review. Krushtalev and Faghri (1994) have shown a one-dimensional model to predict the maximum heat exchange capacity and thermal resistance of a polygonal M.H.P, which was developed from some approximations based in empirical observations. Hopkins et al (1998) compared the one-dimension model to experimental data, obtained in test with trapezoidal micro heat pipes, in order to verify the maximum heat flux. Theoretical prediction of the capillary limitation in the horizontal orientation agreed reasonably with the experimental data.

Ma \& Peterson (1998) investigated the minimum meniscus radius and the heat exchange limit in M.H.P through the conservation laws for momentum and Laplace-Young equation, providing analytical expressions for parameters of interest. Ha \& Peterson (1998) have been developed a model where the differential expression is similar to Bernoulli's equation. An approximate analytical solution for the axial variation of meniscus curvature is presented. Peterson \& Ma (1999) developed a more detailed model for a M.H.P through a $3^{\text {rd }}$ order ordinary differential equation, that is able to estimate the heat transport capacity and the temperature gradients along longitudinal coordinate, as a function of heat flux. However, all these works are 1-D models, just applicable to steady state processes.

The present work is an extension of the work presented by Machado \& Miranda (2001), where a mathematical model and a computational algorithm for simulation of the two-dimensional unsteady heat transfer and phase change processes in an opened square cavity, was proposed. Machado (2003) applied the model to a closed square cavity, representing a flat capillary heat pipe. In this work, a threedimension closed cavity is used to simulate the actual behavior of a capillary heat pipe.

The phase change problem is solved via the interface tracking method proposed by Unverdi and Trygvason (1992), that is an hybrid LagrangeanEulerian method for moving boundaries, and has been employed to simulate fusion/solidification of crystals and metal alloys and bubble formation in nucleate boiling. This method has been successfully extended to three-dimensional problems of phase change (Esmaeeli \& Arpaci, 1998; Shin \& Juric, 2002) and bubble flow (Esmaeeli \& Tryggvason, 1998, 1999; Arruda, 1999).

Preliminary results show the behavior of the computer code for a simple case. The model proposed here may be easily extended to problems with irregular geometries.

\section{PHYSICAL PROBLEM MATHEMATICAL MODEL}

AND

Consider a square cavity, filled with a pure Newtonian fluid with constant properties and isochoric behavior, shown in Fig. (1). Thermodynamic equilibrium is assumed in both phases, liquid and vapor, inside. The lateral and superior sides are thermally insulated.

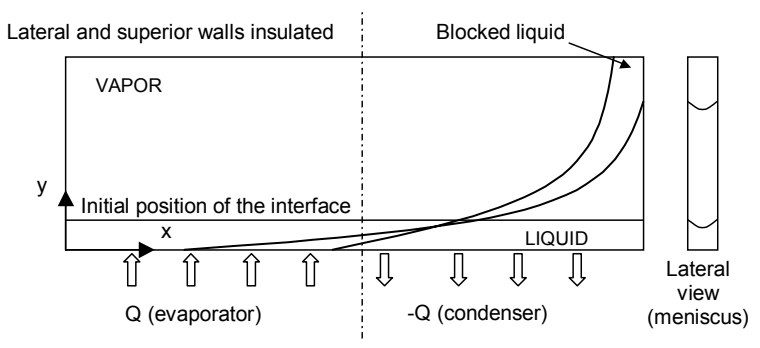

Figure 1. Schematic of geometry and physical process.

The set of equations in dimensionless form used to represent the simple physical problem is written in an appropriate form to be solved by the interface tracking method, as proposed by Juric (1996). Terms in bold are vector quantities.

Continuity equation:

$$
\frac{\partial \rho}{\partial t}+\nabla \cdot \mathbf{w}=0
$$

this form is equivalent to:

$$
\begin{gathered}
\nabla \cdot \mathbf{w}=M \\
M=\int_{A} m \delta\left(\mathbf{x}-\mathbf{x}_{\mathbf{f}}\right) d A \\
m=\left(\frac{\rho_{\mathrm{v}}}{\rho_{1}}\right) \mathbf{V} \cdot \mathbf{n}
\end{gathered}
$$

where $\mathbf{w}=\rho \mathbf{u}$ (mass flux), $\mathbf{V}$ is the interface velocity, $\mathbf{n}$ is the interface normal vector, $m$ is the mass flux through the interface per unit of area and $\delta\left(\mathbf{x}-\mathbf{x}_{\mathbf{f}}\right)$ is a $3-D$ delta function, that is non-zero only at the interface. Subscripts $l$ and $v$ refers to liquid and vapor 
phases, respectively. Tangential effects are neglected, as the fluid properties are assumed constant and uniform in each phase. Therefore, the interface velocity is supposed not having a tangential component. Such assumptions should be verified carefully, in a more detailed model.

Conservation of momentum:

$$
\frac{\partial(\mathbf{w})}{\partial \mathrm{t}}+\nabla \cdot(\mathbf{w u})=-\nabla \mathrm{P}+\frac{1}{\operatorname{Re}} \nabla \cdot \mu\left(\nabla \mathbf{u}+\nabla \mathbf{u}^{\mathrm{T}}\right)+\mathbf{F}
$$

where $\mathrm{P}$ is the pressure and $\mathbf{F}$ is a source term that takes into account the interfacial surface forces:

$$
\mathbf{F}=\int_{\mathrm{A}} \mathbf{f} \delta\left(\mathbf{x}-\mathbf{x}_{\mathbf{f}}\right) \mathrm{dA}
$$

where $\mathbf{x}_{\mathbf{f}}$ is the interface position vector, $\mathbf{f}$ is the interface pressure due to the surface tension: $\mathbf{f}=$ $\left(\kappa_{\mathrm{I}}+\kappa_{\mathrm{II}}\right) . \mathbf{n} / \mathrm{We}$, and $\kappa$ is twice the mean curvature of the interface, and subscripts I and II are related to transversal and longitudinal directions, respectively.

\section{Conservation of energy:}

$$
\frac{\partial(\rho \mathrm{cT})}{\partial \mathrm{t}}+\nabla \cdot(\mathbf{w c T})=\frac{1}{\mathrm{Pe}} \nabla \cdot \mathrm{K} \nabla \mathrm{T}+\mathrm{Q}
$$

where $\mathrm{c}$ is the specific heat at constant pressure, $\mathrm{K}$ is the thermal conductivity and $\mathrm{Q}$ is a source term that takes into account the absorption or liberation of latent heat during phase change:

$$
\mathrm{Q}=\int_{\mathrm{A}} \mathrm{q} \delta\left(\mathbf{x}-\mathbf{x}_{\mathbf{f}}\right) \mathrm{dA}
$$

$q$ is the source term of energy per unit of surface of the interface:

$$
q=\frac{\rho_{1} L}{\rho_{v} L_{0}}(\rho \mathbf{V}-\mathbf{w}) \cdot \mathbf{n}
$$

where $\mathrm{L}_{0}$ is the latent heat of phase change and $\mathrm{L}$ is its corrected form, taking into account different specific heats for each phase:

$$
\mathrm{L}=\mathrm{L}_{0}+\left(\mathrm{c}_{1}-\mathrm{c}_{\mathrm{v}}\right) \mathrm{T}_{\mathrm{v}}
$$

The equations above, with the source terms included, satisfy the jump conditions automatically, assuming a thickless interface.

Although specific volumes are different in the phases, the flow is assumed to be uncompressible, and both densities are considered constant. The total volume occupied for each phase remains constant during the heating. Therefore, the rates of condensation and evaporation must be the same, in order to satisfy the mass conservation within the cavity. During the transient process of heat transfer, the reference pressure inside the cavity rises and yields a correspondent increase of the local saturation temperature in every interface point, until the flow reaches steady state.

The interface is supposed to be at thermal but not thermodynamic equilibrium (which supposes no temperature jump at the interface). This hypothesis added to the Clausius-Clapeyron relation applied to a curved surface, but neglecting the kinect mobility effects yields the temperature condition at the interface as a function of the reference pressure:

$$
\begin{aligned}
& \mathrm{T}_{\mathrm{f}}-\mathrm{T}_{\mathrm{SAT}}-\sigma \kappa+\text { We. } \mathrm{T}_{\mathrm{SAT}}\left(1-\frac{\rho_{1}}{\rho_{\mathrm{v}}}\right)\left(\mathrm{P}_{\mathrm{f}}-\mathrm{P}_{\infty}\right) \\
& +\frac{\rho_{\mathrm{v}}}{\rho_{1}}\left(\frac{\mathrm{c}_{\mathrm{v}}}{\mathrm{c}_{1}}-1\right)\left(\mathrm{T}_{\mathrm{f}}-\mathrm{T}_{\mathrm{SAT}}\right)^{2}+\vartheta(\rho \mathbf{V}-\mathbf{w}) \cdot \mathbf{n}=0
\end{aligned}
$$

where $T_{f}$ is the local interface temperature, $T_{\mathrm{SAT}}$ is the local saturation temperature (from the equation of state), $\mathrm{P}_{\mathrm{f}}$ is the local interface pressure, $\mathrm{P}_{\mathrm{oo}}$ is the reference pressure within the cavity, $\sigma=c_{1} \mathrm{~T}_{\mathrm{o}} \gamma / \rho_{\mathrm{v}} \mathrm{L}_{\mathrm{o}}^{2} \mathrm{y}_{\mathrm{o}}$. $\mathrm{T}_{\mathrm{o}}$ is the reference temperature, $\mathrm{y}_{\mathrm{o}}$ is the reference length and $\gamma$ is the surface tension. Last term includes the non-equilibrium effects in the molecular kinetics: $\vartheta=\rho_{1} c_{1} U_{0} / \rho_{\mathrm{v}} \mathrm{L}_{0} \varphi$, where $\varphi$ is the molecular kinetics coefficient. Small values of $\varphi$ suppress the growth of protrusions in the interface.

The non-dimensional numbers resulting from the mathematical model are: Reynolds number, $\mathrm{Re}=$ $\rho_{1} U_{0} 1 / \mu_{1}$, Peclet number, Pe $=$ Re.Pr, Weber number, $\mathrm{We}=\rho_{\mathrm{l}} \mathrm{U}_{0}{ }^{2} \mathrm{l} / \gamma$ and Froude number, $\mathrm{Fr}=\mathrm{U}_{0}{ }^{2} / \mathrm{G} . \mathrm{y}_{0}(\mathrm{G}$ is the local acceleration of gravity).

\section{METHOD OF SOLUTION}

The moving boundary problem was solved by the Interface Tracking Method, introduced by Unverdi \& Trygvason (1992), and employed by Juric (1996) in the solution of phase change problems. In this method, a fixed uniform Eulerian grid is generated, where the conservation laws are applied over the complete domain. The interface acts as a Lagragean referential, where a moving grid is applied. The instantaneous placement of the interface occurs through the constant remeshing of the moving grid, and each phase is characterized by the Indicator Function, represented by $\mathrm{I}(\mathbf{x}, \mathrm{t})$, which identifies the properties of vapor and liquid phases.

The interface surface is built considering the transversal radius $\left(\mathrm{R}_{\mathrm{I}}\right)$ constant (according to the formal definition of a micro heat pipe), and its longitudinal shape is represented as a parametric curve, $\mathbf{R}(\mathrm{u})$, where the normal and tangent vectors, longitudinal radius $\left(\mathrm{R}_{\mathrm{II}}\right)$ and curvature are extracted from. The interface discretization is shown in Fig (2). The positions of interface points in transversal direction are interpolated by a Lagrange polynomial, 
which allows to obtain the geometric parameters and remeshes the curve, keeping the distance $d$ between curve points within the interval $0.9<\mathrm{d} / \mathrm{h}<1.1$, where $h$ is the distance among the fixed grid points, as shown in Fig. (2).
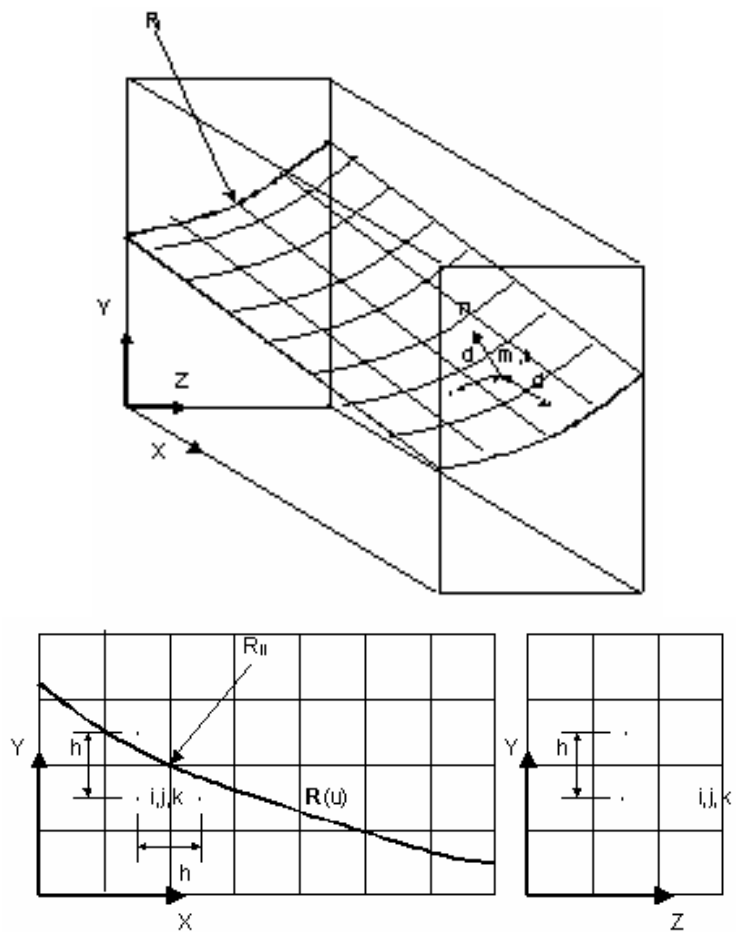

Figure 2. Eulerian and Lagrangean meshes.

The Indicator Function varies from 1 (vapor) to 0 (liquid), and is numerically constructed using the interface curve to determine a source term $\mathbf{G}(\mathbf{x})$. The jump across the interface is distributed over the fixed grid points, yielding a gradient field in the mesh:

$$
\mathbf{G}(\mathbf{x})=\nabla \mathrm{I}=\int_{\mathrm{A}} \mathbf{n} \delta\left(\mathbf{x}-\mathbf{x}_{\mathbf{f}}\right) \mathrm{dA}
$$

which should be zero, except over the interface, as represented by the Dirac Delta Function. However, such representation is not convenient for a discrete number of points. The Distribution Function is used to represent the interface jump. Such Function is a curve similar to a Gaussian one, as shown in Fig (3), and its value depends on the distance $\left|\mathbf{x}_{\mathrm{ij}}-\mathbf{x}_{\mathrm{k}}\right|$ among the Lagrangean and Eulerian points:

$$
\begin{aligned}
& \mathrm{D}_{\mathrm{i}, \mathrm{j}, \mathrm{k}}\left(\mathbf{x}_{\mathrm{m}, \mathrm{n}}\right)= \\
& \frac{\mathrm{f}\left[\left(\mathrm{x}_{\mathrm{m}, \mathrm{n}}-\mathrm{x}_{\mathrm{i}}\right) / \mathrm{h}\right] \cdot \mathrm{f}\left[\left(\mathrm{y}_{\mathrm{m}, \mathrm{n}}-\mathrm{y}_{\mathrm{j}}\right) / \mathrm{h}\right] \cdot \mathrm{f}\left[\left(\mathrm{z}_{\mathrm{m}, \mathrm{n}}-\mathrm{z}_{\mathrm{k}}\right) / \mathrm{h}\right]}{\mathrm{h}^{3}}
\end{aligned}
$$

where $D_{i j}$ is the Distribution Function for a point $k$ in the Lagrangian mesh in relation to a Eulerian point. One should note that increasing $\mathrm{h}$, the interface becomes thicker. The function $f$ is the probability distribution, related to the distance $\mathrm{h}$ as:

$$
f(x)= \begin{cases}f_{1}(x) & \text { if }|x| \leq 1 \\ 1 / 2-f_{1}(2-|x|) & \text { if } 1<|x|<2 \\ 0 & \text { if }|x| \geq 2\end{cases}
$$

$$
f_{1}(x)=\frac{3-2 \cdot|x|+\sqrt{1+4 \cdot|x|-4 x^{2}}}{8}
$$

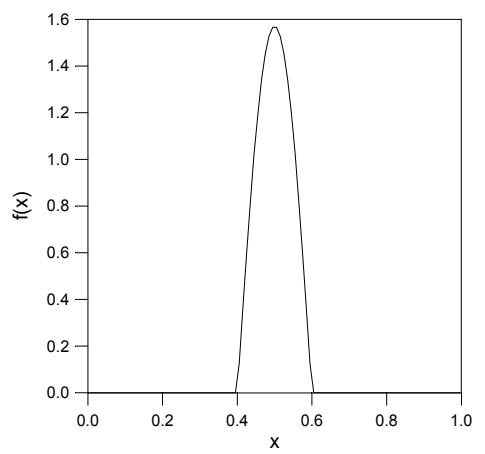

Figure 3. Probabilistic distribution profile - $f(x)$.

The vector $\mathbf{G}(\mathbf{x})$ is obtained from:

$$
\mathbf{G}_{\mathbf{i}, \mathbf{j}, \mathbf{k}}=\sum_{\mathrm{m}, \mathrm{n}} \mathrm{D}_{\mathrm{i}, \mathrm{j}, \mathrm{k}}\left(\mathbf{x}_{\mathbf{m}, \mathbf{n}}\right) \cdot \mathbf{n}\left(\mathbf{x}_{\mathbf{m}, \mathbf{n}}\right) \cdot \mathrm{dS}\left(\mathbf{x}_{\mathbf{m}, \mathbf{n}}\right)
$$

where $\mathrm{dS}\left(\mathrm{x}_{\mathrm{m}, \mathrm{n}}\right)$ is the surface element, shown in Fig.(4), and given as dS = dx'.dy'.

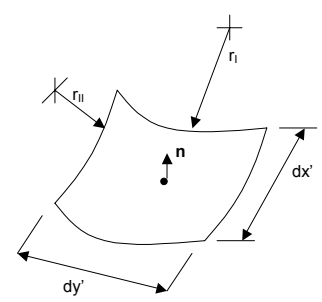

Figure 4. Surface element, dS.

The divergence of the gradient field is found by numerical derivation of Poison's equation:

$$
\nabla^{2} \mathrm{I}=\nabla \cdot \mathbf{G}
$$

Despite of being considered constants in each phase, the properties inside the domain must be treated as variable in the formulation. A generic property $\phi(\rho, \mu$, c or K ) is expressed as:

$$
\phi(\mathbf{x})=\phi_{1}+\left(\phi_{\mathrm{v}}-\phi_{1}\right) \mathrm{I}(\mathbf{x}, \mathrm{t})
$$

The coupling between the moving mesh and the fixed grid is done at each time step, through the Distribution Function, that represents the source terms in the balance equations and interpolates the fields with infinitesimal discontinuities into a finite 
thick region at the interface. The momentum equation at the interface surface becomes:

$$
\frac{\partial \mathrm{I}}{\partial \mathrm{t}}=-\mathbf{V} \cdot \nabla \mathrm{I}
$$

The initial interface shape is first specified and then the Indicator Function is constructed. The property and temperature fields are determined. The iterative process targets the reference pressure and its correspondent interface velocity at each time step. The steps to be followed are:

1. Using the current value of $\mathbf{V}$, the interface points are transported to a new position, calculated explicitly through the equation $\mathbf{V}^{\mathrm{n}}=\left(\mathrm{d} \mathbf{x}_{\mathrm{f}} / \mathrm{dt}\right) \cdot \mathbf{n}$;

2. Density and specific heat are calculated at the new interface position;

3. Surface tension $\mathbf{f}$ is calculated and distributed into the fixed grid;

4. In the first iteration, reference pressure is estimated. After that, its value is corrected according to the mass flux residual.

5. $\mathbf{V}^{\mathrm{n}+1}$ is estimated via Newton iterations, using a numerical relaxation schedule, and used to calculate the mass flux m crossing the interface. The interface mass flux is distributed in the fixed gird;

6. According to boundary conditions, $\mathbf{w}, \mathbf{u}$ e $\mathrm{P}$ are obtained from Eqs. (2) and (5);

7. Density found in step 2 and flux $\mathbf{w}$ are interpolated via distribution function in order to find the mass flux $\mathbf{w}_{\mathrm{f}}$ and density $\rho_{\mathrm{f}}$ at the interface;

8. Heat flux q is calculated through Eq. (9) and distributed into the fixed grid;

9. According to boundary conditions, energy equation - Eq. (7) is used to obtain the temperature at time $n+1$;

10. Temperature and pressure are interpolated to find $\mathrm{T}_{\mathrm{f}}$ and $\mathrm{P}_{\mathrm{f}}$ at the interface;

11. The equilibrium condition is tested. If it is not satisfied, a new estimate for $\mathbf{V}^{\mathrm{n}+1}$ is calculated and returns to step 5 .

12. The mass flux residual is calculated, and if it is lower than the reached tolerance, the fields of viscosity and conductivity are updated for the new position, advance one step time, otherwise returns to step 4 .

The convergence criterion used in step 11 is the residual in Eq. (11). Once it has reached the desired tolerance, convergence for interface velocity is assumed. Otherwise, the velocity is corrected via Newton Iterations, given as:

$$
\mathbf{V}^{\mathrm{n}+1}=\mathbf{V}^{\mathrm{n}}-\omega \cdot \mathbf{R}(\mathrm{T})
$$

where $\omega$ is a constant and $R(T)$ is the residual for the temperature jump condition at the interface. Iteration are repeated until $\mathrm{R}(\mathrm{T})$ in every point become smaller than the tolerance. The optimum value for $\omega$ is found by tentative, at the beginning of the calculation.

A similar schedule is used to obtain the reference pressure, where the mass flux residual is the algebraic summation of the source term M, Eq. (3), along the interface.

\section{RESULTS AND DISCUSSION}

FORTRAN language was used to construct the numerical code and it runs in a PC microcomputer. The transport equations were solved in a regular displaced mesh, via finite volume method, where the $\mathrm{P}-\mathrm{V}$ coupling schedule used was the PRIME algorithm, and the discretization was done under the FTCS schedule.

\section{Validation of the numerical method}

The numerical results were validated by a variation of the one-dimensional Stefan problem, within a $0<\mathrm{x}<1$ domain, with a constant heat flux at $x=0$, and constant temperature at $x=1$. The initial interface position is $\mathrm{x}_{\mathrm{f}}=0.5$, with the liquid phase at $\mathrm{x}<0.5$ and vapor phase at $\mathrm{x}>0.5$. Both phases are at saturation temperature when a heat flux $q$ is imposed at $\mathrm{x}=0$. The values used were $\mathrm{K}_{\mathrm{v}} / \mathrm{K}_{1}=0.33, \rho_{\mathrm{v}} / \rho_{\mathrm{l}}=$ $0.5, \mathrm{c}_{\mathrm{v}} / \mathrm{c}_{1}=0.5, \mathrm{q}=0.1, \mathrm{~T}_{\mathrm{sat}}=1, \mathrm{Pe}=0.04, \mathrm{Re}=0.08$ and $\mathrm{We}=0$.

The results for the interface velocity are compared to those obtained via the Generalized Integral Transform Technique - GITT (Cotta et al, 1998). In Fig. (5), good agreement between both methods of solution is observed. The numerical solution improves as the number of points of Langragian mesh (ni) increases. This improvement is remarkable at the initial and steady state times. In this case, the number of points of the Eulerian mesh also increases, due the meshes coupling by the Distribution Function.

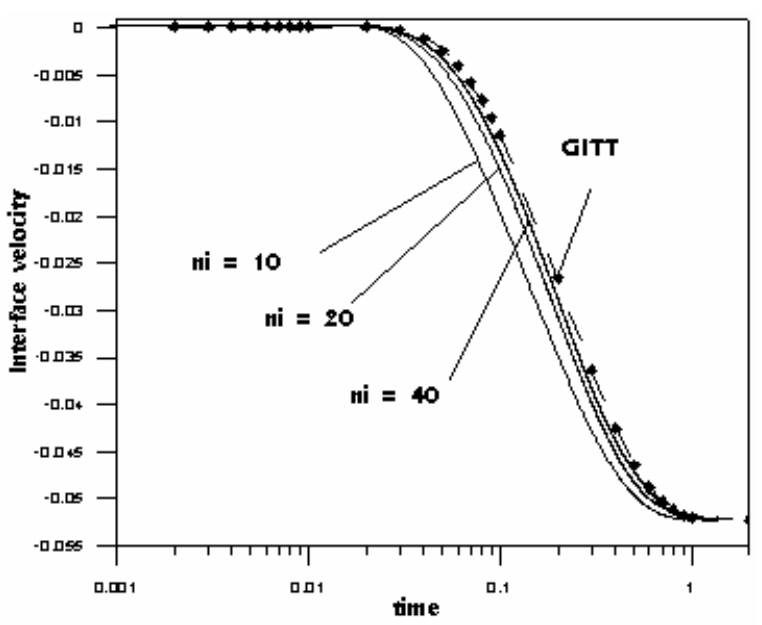

Figure 5. Comparison between Interface Tracking Method and GITT. 
In Fig. (6) the effect of the interface thickness may be observed in the profiles of pressure and vertical velocity component. The thickness reduces as the number of points used increases. With ni $=10$, interface thickness occupies a considerable fraction of the domain. With $\mathrm{ni}=40$, the discontinuity at the interface is represented with a good precision. When few points are used in the Lagragian mesh, the results out of the interface are very close to the exact values, that are reached when $\mathrm{ni}=20$.

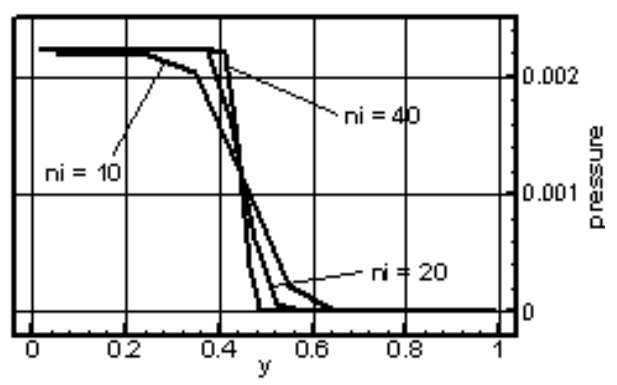

(a) Pressure profile

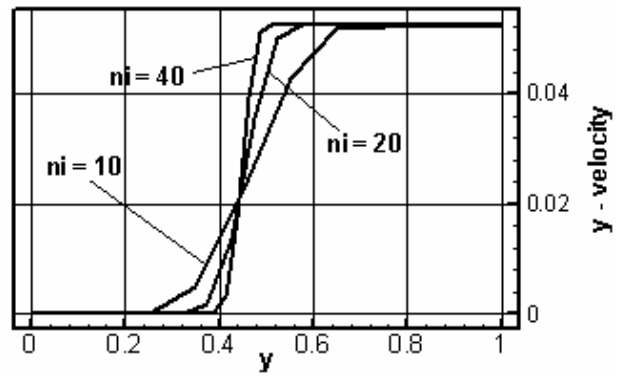

(b) Vertical velocity profile

Figure 6. Solutions according with the number of points used in the Lagrangean mesh (ni).

\section{Results for a square cavity}

Preliminary results for 3-D unsteady simulation were obtained for a flat square cavity representing a micro heat pipe, considering the effects of gravity and surface tension. The domain considered is the region defined as $0<\mathrm{x}<1$ and $0<\mathrm{y}<1$ and $0<\mathrm{z}<$ 0.5 , under prescribed temperatures in the inferior wall, $\mathrm{T}_{\mathrm{w}}=11$, in $0<\mathrm{x}<0.25$ and $\mathrm{T}_{\mathrm{w}}=-9$, in $0.75<\mathrm{x}$ $<1.0$, considering $\mathrm{t}=1$ the dimensionless saturation temperature at the initial conditions. In the intermediary section, $0.25<\mathrm{x}<0.75$, the wall is thermally insulated, $\mathrm{q}_{\mathrm{w}}=0$. Lateral and superior walls are also insulated. The values used in the test case, for the physical parameters, were: $\mathrm{K}_{\mathrm{v}} / \mathrm{K}_{1}=0.5$, $\rho_{\mathrm{v}} / \rho_{1}=0,9 \mathrm{c}_{\mathrm{v}} / \mathrm{c}_{1}=0.5, \mu_{\mathrm{v}} / \mu_{1}=0.5, \mathrm{Pe}=2, \mathrm{Re}=1, \mathrm{We}$ $=3.96, \mathrm{Fr}=3.92, \sigma=0.99$ e $\vartheta=0.4$, and the interface is placed at $\mathrm{y}=0.5$ at $\mathrm{t}=0$. A $10 \times 10 \times 5$ points grid was used.

The schedule for correcting the reference pressure was tested using a simplified second order polynomial as the equation of state:

$$
\mathrm{T}_{\mathrm{SAT}}=0.1 \cdot\left(\mathrm{P}+\mathrm{P}_{\infty}\right)^{2}+\mathrm{T}_{\mathrm{v} 0}
$$

where $T_{\mathrm{v} 0}$ is the saturation temperature for the initial reference pressure, equal to 1 . For this case, the initial value for the reference pressure is taken to zero.

Figure (7) shows the variation of the reference pressure along the time for several values of the meniscus radius, and its comparison with the results for the two-dimensional case. Reference pressure varies strongly with the radius. When $\mathrm{R}<1.2$, steady state is not reached before the interface touch the superior wall (when the calculations are stopped). The curve for $\mathrm{R}=10^{10}$ (infinite) is similar to the 2-D simulation, but the reference pressure is twice that for two-dimensional calculations. The difference between the infinite radius and 2-d cases is that the first is a 3-D case, but the interface is plane in the $\mathrm{z}$ direction. Interface shape at $\mathrm{t}=2.2$ (were the steady state has been reached for infinite radius), Fig. (8), presents little variation, but its right extremity seems to be more stable than the $2-\mathrm{D}$ interface for $\mathrm{t}=1.1$.

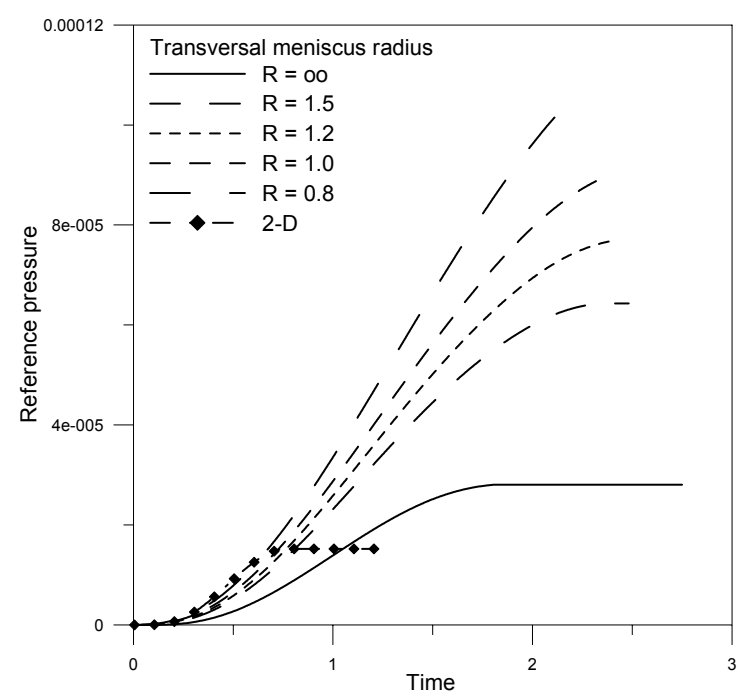

Figure 7. Reference pressure variation with time.

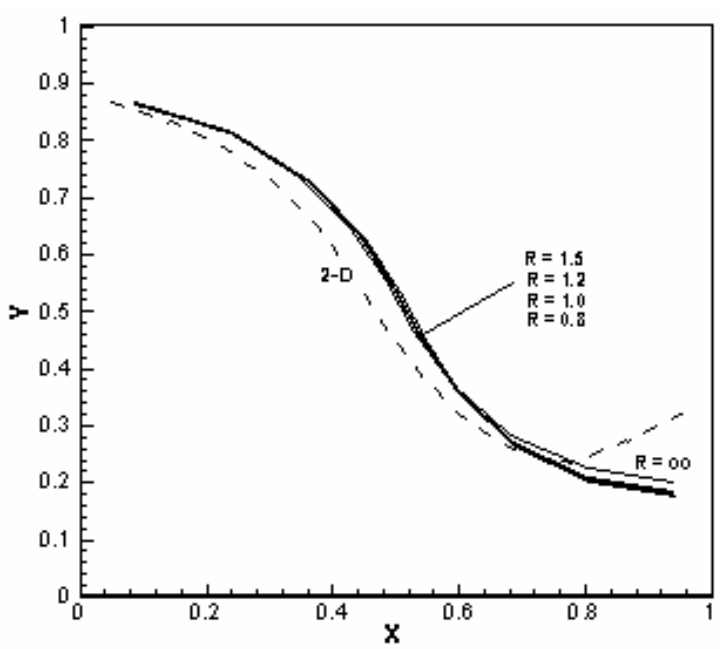

Figure 8. Interface shape at $\mathrm{t}=2.2(\mathrm{t}=1.1$ for $2-\mathrm{D}$ case). 
In Fig. (9), the streamlines for $\mathrm{R}=\mathrm{oo}$ and $\mathrm{R}=1.2$ are similar. The interface perturbation in the $2-\mathrm{D}$ case appears as a displacement of the center of recirculation within the cavity. Figure (10) shows the 3 -D view of the flow filed for $\mathrm{R}=0.8$ and infinite. There is no apparent influence of the transversal meniscus radius in flow patterns. Transversal flow is not observed, even for $\mathrm{R}=0.8$.

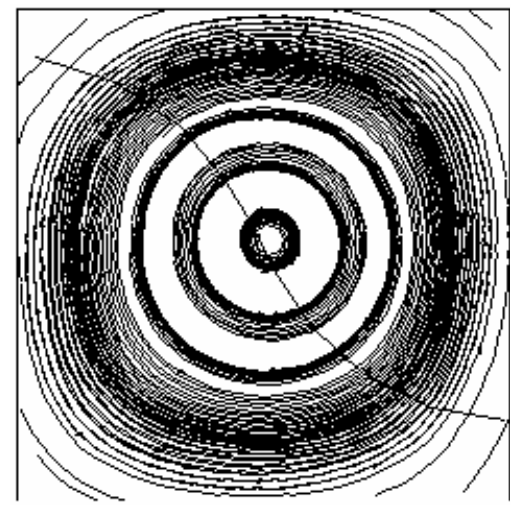

a) $\mathrm{R}=\mathrm{oo}$

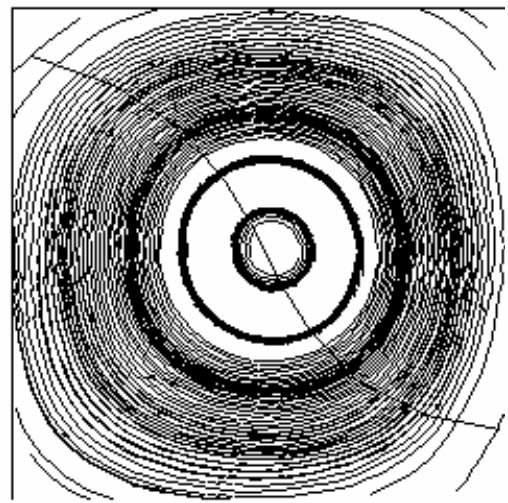

b) $\mathrm{R}=1.2$

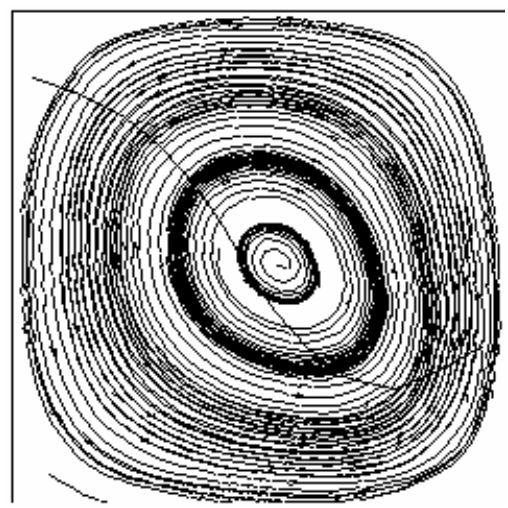

c) 2-D

Figure 9. Streamline in $\mathrm{z}=0.25$ for the $3-\mathrm{D}$ simulation and 2-D simulation, after steady state has been reached.
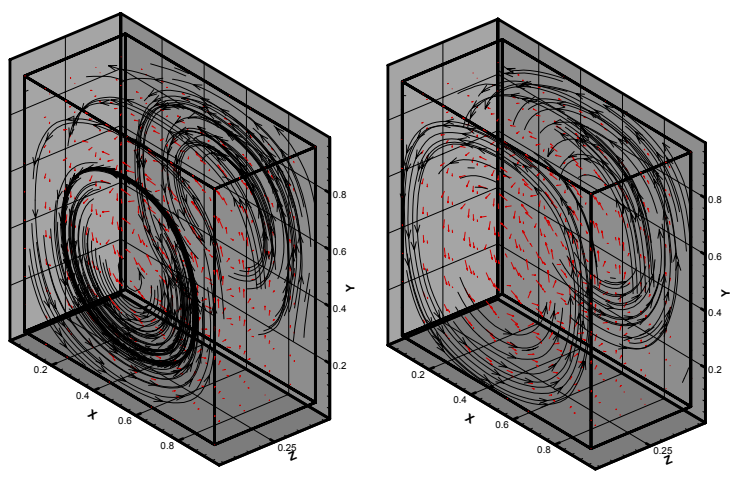

Figure 10. Three-dimensional view of streamlines and velocity vectors at $t=2.2$, for $R=0.8$ and infinite.

In Fig. (11), the evolution of interface position with the time (starting as an horizontal line) is shown for $\mathrm{R}=\infty$, demonstrating the good behavior of the grid reconstruction schedule. At $\mathrm{t}=1$, the interface displacement is still small, although it indicates the tendency for its final profile. Steady state profile is shown at $\mathrm{t}=2.2$.

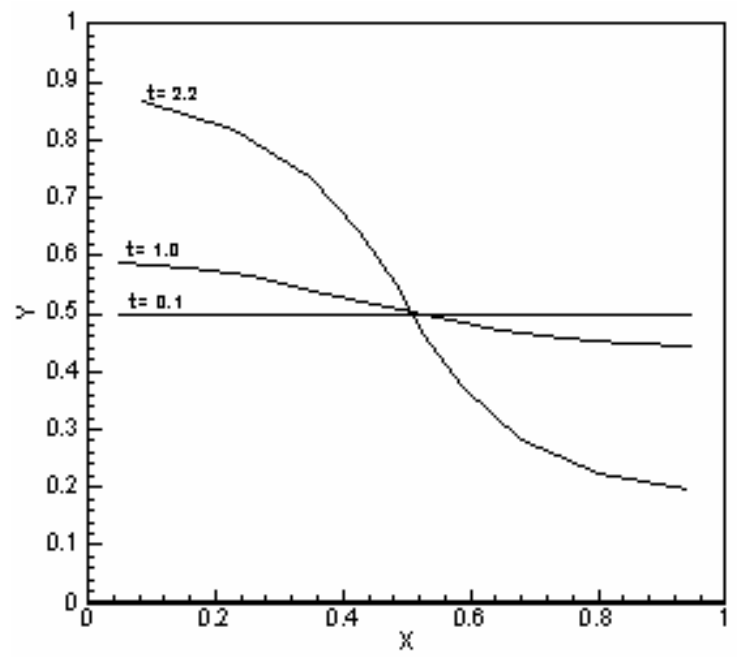

Figure 11. Evolution of interface along the time for $\mathrm{R}=\infty$.

In Fig. (12), interface velocity, temperature and pressure over the interface surface are shown. Velocity and temperature distributions are homogeny and similar for $\mathrm{R}=0.8$ and infinite. Interface temperature varies according to the jumping conditions, from Eq. (12). Variation of pressure is influenced by the increase of the hydrostatic pressure and transversal radius of curvature of the meniscus. Such influence yields the negative values observed when $\mathrm{R}=0.8$. 

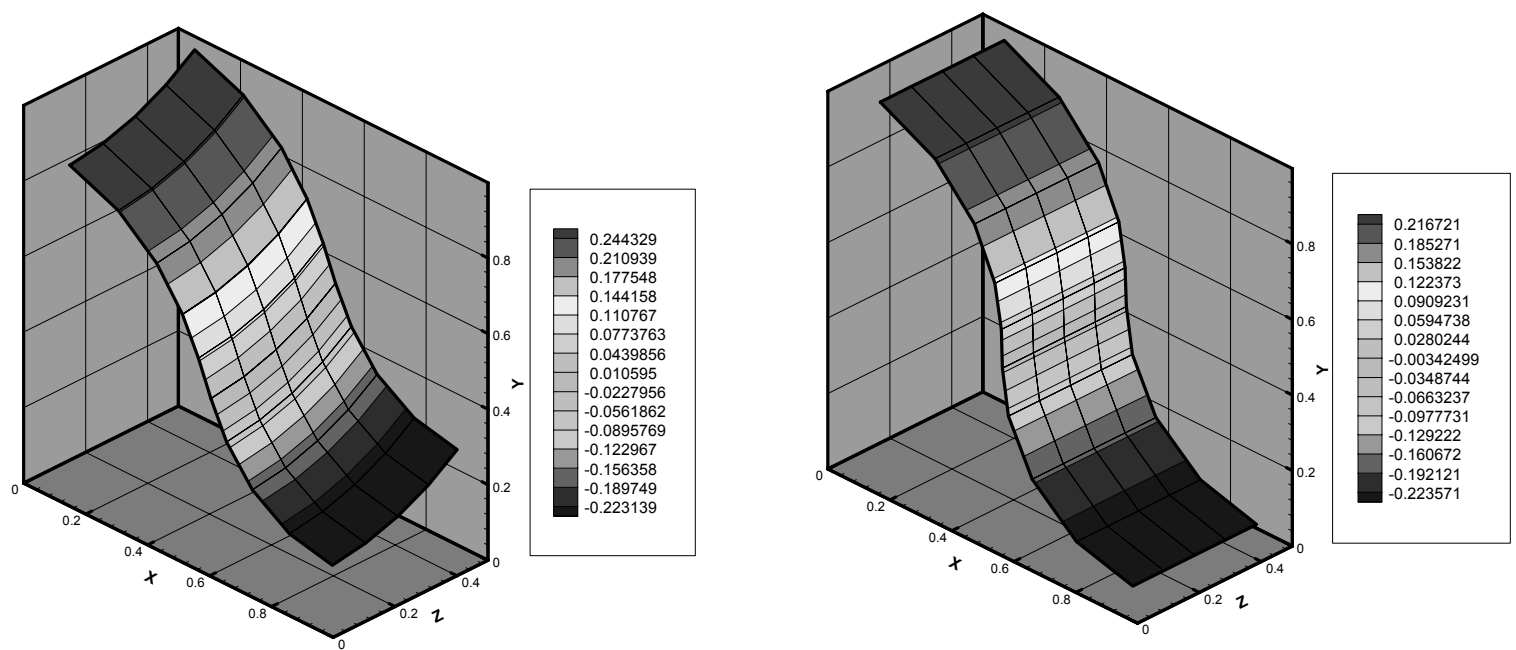

(a) Interface velocity
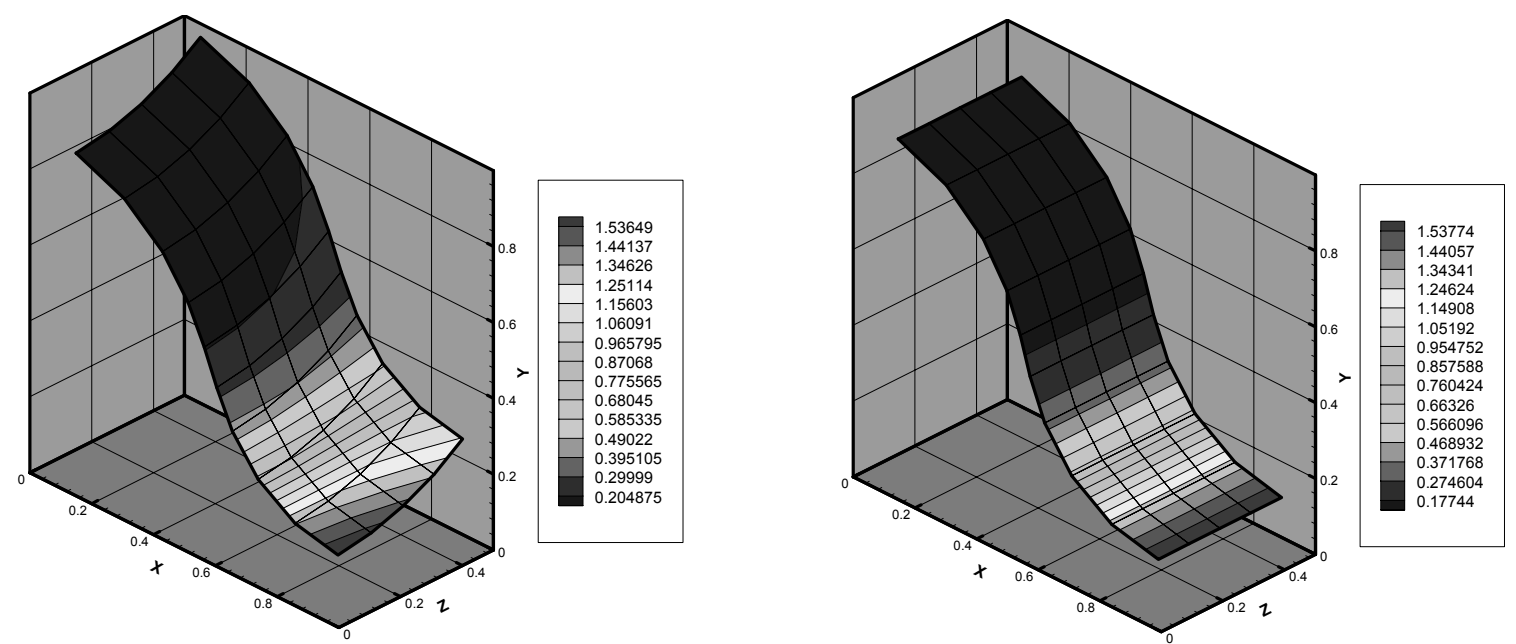

(b) Interface temperature

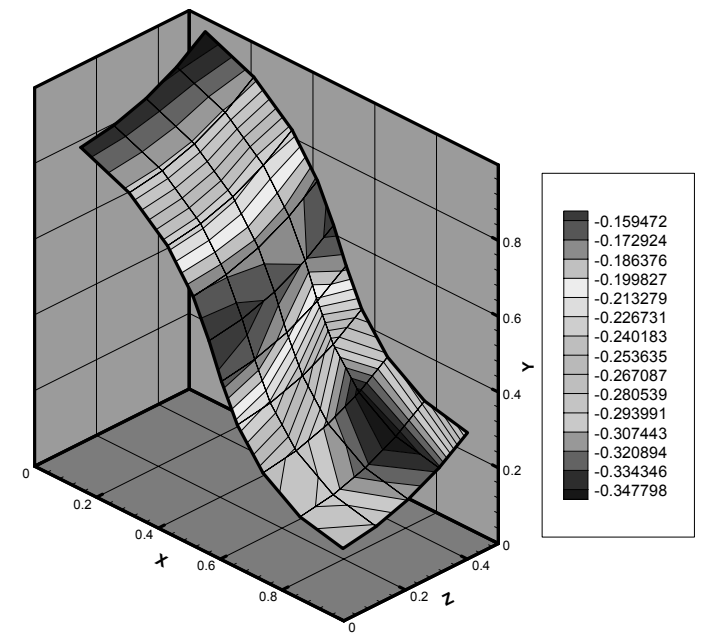

c) Interface pressure

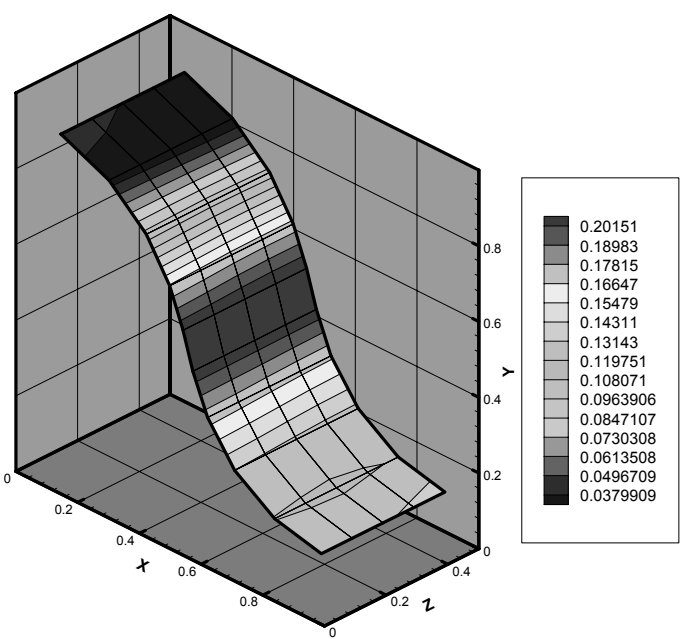

Figure 12. Interface velocity, temperature and pressure and temperature at $=2.2$, for $\mathrm{R}=0.8$ and infinite. 
Table (1) shows the values for dimensionless thermal resistance at $t=2.2$ for several values of transversal meniscus radius. The values rises with meniscus radius, what may indicate that thinner heat pipes would be relatively more effective for the same fluid, operating under same conditions. As it would be expected the value closest to the two-dimensional case is that for infinite radius, when the interface has no transversal variation in its shape.

Table 1. Dimensionless thermal resistance as a function of transversal meniscus radius at $\mathrm{t}=2.2$, compared with two-dimensional result at $\mathrm{t}=1.1$.

\begin{tabular}{|l|c|l|}
\hline \multicolumn{1}{|c|}{$\begin{array}{c}\text { Meniscus } \\
\text { Radius }\end{array}$} & $\begin{array}{c}\text { Thermal } \\
\text { Resistance }\end{array}$ & \multicolumn{1}{c|}{ Obs. } \\
\hline Infinite & 0.58096 & \\
\hline 1.5 & 0.58369 & \\
\hline 1.2 & 0.58576 & \\
\hline 1.0 & 0.58887 & $\begin{array}{l}\text { Steady state not } \\
\text { reached }\end{array}$ \\
\hline 0.8 & 0.59593 & $\begin{array}{l}\text { Steady state not } \\
\text { reached }\end{array}$ \\
\hline 2-D at $\mathrm{t}=1.1$ & 0.58115 & \\
\hline
\end{tabular}

\section{CONCLUSIONS}

In this work, the Interface Tracking Method was employed to simulate the unsteady 3-D heat transfer and phase change process inside a flat capillary heat pipe, represented by a square closed cavity. The code was validated comparing its results to an exact solution of a one-dimensional problem, demonstrating its capacity to represent the interface discontinuity. Such representation improves as more points are added to the Lagrangean mesh.

The complete physical model was tested, considering a constant transversal meniscus radius, and the method shown to be able to represent the physical process end capture the differences due to the variation of the transversal meniscus radius. The radius rising yields a delay in reaching the steady state and increases thermal resistance and reference pressure. When compared with two-dimensional results, the flow patterns for 3-D simulation seem to be more stable, and the reference pressure for steady state gets higher.

Although a rough mesh was employed, the results present physical coherence and are qualitative close to that expected for a heat pipe. Complete test for fluids and dimensions found in feasible heat pipes in refined meshes are necessary for comparison to the one-dimensional models and experimental data available in literature, in order to verify the accuracy of the physical model. The methodology described in this work can be extended easily to irregular geometries.

\section{ACKNOWLEDGEMENTS}

The author would like to acknowledge to FAPESP for the financial support during this work.

\section{REFERENCES}

Arruda, J. M., 1999, Simulação Numérica dos Processos de transporte, Deformação e Captura de Interfaces tridimensionais, Msc. Thesys, Universidade Federal de Uberlândia, Uberlândia Brazil.

Cotta, R. M., 1998, The Integral Transform Method in Thermal and Fluids Science and Engineering, Begell House.

Duncan, A. B., and Peterson, G. P., 1994, Review of Microscale Heat Transfer, Applied Mechanics Review, Vol. 47, No.. 9, pp. 397-428.

Esmaeeli, A., and Arpaci, V., 1998, Numerical Modeling of Three-dimensional Fluid Flow with Phase Change, in: Proc. $4^{\text {th }}$ Microgravity Fluid Physics \& Transport Phenomena Conference, Cleveland, Ohio, pp. 546-551.

Esmaeeli, A., and Tryggvason, G., 1998, Direct Numerical simulation of Bubble Flows - Part 1 Low Reynolds Number Arrays, J. Fluid Mechanics, Vol. 377, pp. 313-345.

Esmaeeli, A., and Tryggvason, G., 1999, Direct Numerical simulation of Bubble Flows - Part 2 Moderate Reynolds Number Arrays, J. Fluid Mechanics, Vol. 385, pp. 325-358.

Faghri, A., 1995, Heat Pipe Science and Technology, Taylor\&Francis.

Hopkins, R., Faghri, A., and Khrustalev, D., 1999, Flat Miniature Heat Pipes with Micro Capillary Grooves, J. Heat Transfer, Vol. 121, pp. 102-109.

Huang, J., Karthikeyan, M., Plawsky, J., Wayner Jr., P.C., 1998, Constrained Vapor Bubble, in: Proc. $4^{\text {th }}$ Microgravity Fluid Physics \& Transport Phenomena Conference, Cleveland, Ohio, pp. 155159.

Juric, D., 1996, Computations of Phase Change, PhD. Thesis, University of Michigan.

Juric, D. and Tryggvason, G., 1998, Computations of Boiling flows, Int. J. of Multiphase Flow, Vol. 24, No. 3, pp 387-410.

Khruhstalev, D., and Faghri, A., 1994, Thermal Analysis of a Micro Heat Pipe, Journal of Heat Transfer, Vol. 116, pp. 189-198.

Ma, H. B., and Peterson, G. P., 1998, The Minimum Meniscus Radius and Capillary Heat Transport Limit in Micro Heat Pipes, Journal Heat Transfer, Vol. 120, pp. 227-233.

Machado, H. A., and Miranda, R. F., 2001, Simulation of Micro Heat Pipes Using an Interface Tracking Method, in: Proceedings of the XVI COBEM - Brazilian Congress of Mechanical Engineering, Uberlândia, Brazil.

Machado, H. A., 2003, Simulation of a Flat Capillary Heat Pipe Using an Interface Tracking 
Method, in: Proceedings of the XVII COBEM Brazilian Congress of Mechanical Engineering, São Paulo, Brazil.

Peterson, G. P., 1994, An Introduction to Heat Pipes - Modeling, Testing and Applications, John Wiley \& Sons.

Peterson, G. P., Ha, J. M., 1998, Capillary Performance of Evaporating Flow in Micro Grooves: An Approximate analytical Approach and Experimental Investigation, Journal Heat Transfer, Vol. 120, pp. 743-751.

Peterson, G. P., and Ma, H. B., 1999, Temperature Response of Heat Transport in a Micro Heat Pipe, Journal Heat Transfer, Vol. 121, pp. 438445.

Prat, D. M., Brown, and J. R., Hallinan, K. P., 1998, Thermocapillary Effects on the Stability of a Heated, Curved Meniscus, Journal Heat Transfer, Vol. 120, pp. 220-226.

Ravigururajan, T. S., 1998, Impact of Channel Geometry on Two-Phase Flow Heat Transfer Characteristics of Refrigerants in Microchannel Heat Exchangers, Journal Heat Transfer, Vol. 120, pp. 485-491.

Shin, S., and Juric, D., 2002, Modeling threedimensional Multiphase flow Using a Level contour Reconstruction Method for Front Tracking Without Connectivity, J. Comp. Physics, Vol. 180, pp. 427470.

Sun, K. H., and Tien, C. L., 1972, Single Conduction Model for Theoretical Steady-State Heat Pipe Performance, AIAA Journal, Vol. 10, pp. 10511057.

Unverdi, S. O., and Tryggvason, G., 1992, A Front-Tracking Method for Viscous, Incompressible, Multi-fluid Flows, Journal of Computational Physics, Vol. 100, pp. 25-37.

Received: February 05, 2006

Revised: March 05, 2006

Accepted: April 05, 2006 